\title{
A importância da interação entre odontopediatrias e pediatrias no manejo de dentes natais e neonatais
}

\author{
The importance of interaction of pediatric dentists and pediatricians \\ in the management of natal and neonatal teeth
}

Michele Baffi Diniz¹, Juliana Oliveira Gondim¹, Cyneu Aguiar Pansani², Fabio Cesar B. de Abreu-e-Lima²

\section{RESUMO}

Objetivo: Apresentar revisão de literatura sobre dentes natais e neonatais, abordando características clínicas, fatores etiológicos, medidas terapêuticas e a importância do conhecimento desta anomalia, por odontopediatras e pediatras.

Fontes de dados: Foram selecionados os artigos mais relevantes sobre o tema, desde 1950 até 2006, pesquisados no Medline e na Bibliografia Brasileira em Odontologia (BBO), além de livros de pertinentes.

Síntese dos dados: Os dentes natais e neonatais consistem em uma anomalia de erupção, sendo caracterizados por seu irrompimento na cavidade oral durante o período intra-uterino ou no primeiro mês de vida respectivamente, podendo fazer parte da dentição decídua normal ou supranumerária. Esses dentes, em geral, apresentam bordos cortantes e podem estar relacionados ao aparecimento de ulcerações na base da língua do bebê e/ou no seio materno, comprometendo a amamentação. A fraca implantação óssea desses dentes favorece sua grande mobilidade, tornando-se, assim, um fator de risco à sua aspiração ou deglutição pela criança. A abordagem terapêutica depende da dentição à qual pertence o dente e dos possíveis problemas que este pode causar à saúde da criança ou da mãe.

Conclusões: $O$ conhecimento sobre as características clínicas e os possíveis distúrbios aos quais os dentes natais e neonatais estão relacionados por odontopediatras e pediatras possibilita a interação necessária para o diagnóstico precoce e a abordagem integral da criança.

Palavras-chave: dente decíduo; dentes natais; Odontopediatria; Pediatria.

\section{ABSTRACT}

Objective: To review available data about natal and neonatal teeth, emphasizing clinical characteristics, etiological factors, treatment, and the importance of the knowledge about this anomaly by pediatric dentists and pediatricians.

Data sources: The most relevant articles published on the subject were selected, from 1950 to 2006, browsed on Medline, Brazilian Bibliografy of Odontology (BBO) and Dentistry books.

Data synthesis: Natal and neonatal teeth are considered eruption anomalies, characterized by eruption into oral cavity during the intrauterine period or during the first month after birth, respectively, being part of the regular deciduous or the supernumerary dentitions. As these teeth usually present cutting edges, they can be related to traumatic injury to newborn's tongue and/or to mother's nipples, compromising breastfeeding. In general, the weak attachment of these teeth to alveolar bone favors a high degree of mobility, which exposes the infant to the risk of aspiration or swallowing them. The management depends on which dentition these teeth belong and/or on complications that may affect either infant's or mother's health.

Conclusions: The knowledge about the clinical characteristics and the possible complications related to natal and neonatal teeth by pediatric dentists and pediatricians provides the necessary interaction to early diagnosis and infant general management.

Key-words: tooth, deciduous; natal teeth; Pediatric Dentistry; Pediatrics

Endereço para correspondência:

Fabio Cesar B. de Abreu-e-Lima

Departamento de Clínica Infantil da Faculdade de Odontologia de Araraquara da Unesp

Rua Humaitá, 1.680 - Centro

CEP 14801-903 - Araraquara/SP

E-mail: michelebdiniz@foar.unesp.br

Recebido em: 25/6/2007

Aprovado em: 3/10/2007 


\section{Introdução}

O desenvolvimento dos dentes decíduos inicia-se a partir da sexta semana de vida intra-uterina, quando ocorre a proliferação do epitélio que originará o órgão dental. A mineralização ocorre nesse período e, ao nascimento, 3/5 do esmalte coronário dos incisivos inferiores apresenta-se formado ${ }^{(1)}$. Os incisivos centrais inferiores decíduos são os primeiros dentes a irromper na cavidade oral, por volta dos seis meses de vida da criança.

Entretanto, têm sido relatados na literatura casos de crianças que já nascem com dentes erupcionados na cavidade oral ou que erupcionam no primeiro mês de vida, denominados dentes natais e neonatais, respectivamente ${ }^{(2,3)}$. Esses dentes podem fazer parte da dentição decídua normal ou serem dentes supranumerários, são mais freqüentes na região anterior da mandíbula e os incisivos inferiores correspondem aos dentes mais comumente envolvidos ${ }^{(4,5)}$.

Essa anomalia de erupção acarreta preocupações tanto para os pais das crianças como para os profissionais da área de saúde, uma vez que os dentes natais e neonatais, além de apresentarem acentuada mobilidade ${ }^{(6)}$, podem ser deglutidos ou aspirados pela criança ${ }^{(7)} \mathrm{ou}$, ainda, causar traumas no mamilo do seio materno e ulcerações no ventre da língua do recém-nascido, dificultando o aleitamento ${ }^{(3,8)}$.

O objetivo deste trabalho foi analisar os dados da literatura referentes aos dentes natais e neonatais, abordando características clínicas, fatores etiológicos, medidas terapêuticas e a importância do conhecimento desta anomalia, tanto pelos odontopediatras como pelos pediatras - profissionais da área de saúde que mais acompanham o desenvolvimento da criança.

\section{Revisão da literatura}

\section{Histórico}

Os dentes natais e neonatais são de ocorrência rara e têm sido observados e relatados como "incrustações cuneiformes" desde $59 \mathrm{AC}^{(9)}$. No passado, acreditava-se estarem relacionados a mitos folclóricos e superstições ${ }^{(10)}$. De acordo com a cultura local, o seu aparecimento indicaria uma proteção e benção divina ou, para outros, um carma ou um futuro não promissor para o bebêt $\hat{e}^{(3,11,12)}$.

Diversos termos foram atribuídos para designar a anomalia, tais como dente fetal, dente congênito, dente pré-decíduo e dentição precoce ${ }^{(4,5)}$. Entretanto, a denominação mais empregada atualmente é "dente natal" e "dente neonatal" proposta por Massler e Savara ${ }^{(13)}$, baseada na época de erupção dentária, ou seja, dentes natais são aqueles observados na cavidade oral ao nascimento e dentes neonatais, aqueles que erupcionam durante os primeiros 30 dias de vida.

\section{Epidemiologia}

A prevalência dos dentes natais e neonatais varia entre $1: 800$ a 1:3.000 $0^{(9,10,13-15)}$. Tal variação depende das diferentes populações estudadas e dos métodos empregados em cada estudo. Em pesquisa realizada por de Almeida e Gomide ${ }^{(16)}$, foi relatada alta prevalência em pacientes fissurados $(10,6 \%$ com fissuras palatinas bilaterais completas e $2,02 \%$ com fissuras palatinas unilaterais completas), fato atribuído à presença das fissuras alveolares e à posição superficial do germe dentário nestes pacientes.

Muitos autores relataram predileção pelo sexo feminino ${ }^{(9,14,17,18)}$; entretanto, Gorlin $e t$ a $l^{(19)}$ não observaram diferença na prevalência entre os sexos. Os dentes natais são encontrados com maior freqüência, quando comparados aos dentes neonatais, na proporção de $3: 1^{(5,10,13,17)}$. Aproximadamente $90 \%$ dos dentes natais e neonatais fazem parte da dentição decídua, enquanto os $10 \%$ restantes são supranumerários ${ }^{(9,10,13,15)}$.

Segundo Cunha et al ${ }^{(3)}$ e Leung e Robson ${ }^{(15)}$, os incisivos centrais inferiores são os mais acometidos, devido ao fato de que esses dentes comumente são os primeiros a erupcionar na cavidade oral da criança. De acordo com Bodenhoff e Gorlin $^{(10)}$, a incidência dessa anomalia em incisivos centrais inferiores é de $85 \%$, seguidos pelos incisivos centrais superiores $(11 \%)$, caninos e molares inferiores $(3 \%)$ e caninos e molares superiores $(1 \%)$.

\section{Etiologia}

De acordo com a literatura, alguns fatores podem estar relacionados ao aparecimento de dentes natais e neonatais na cavidade oral, tais como a posição superficial do germe dentário ${ }^{(20,21)}$, infecção $0^{(9)}$, efeitos da sífilis congênita ${ }^{(13)}$, erupção acelerada por pico febril ou estímulo hormonal ${ }^{(22,23)}$, hereditariedade $^{(5,21,24,25)}$ e deficiências nutricionais ${ }^{(9,14)}$. Entretanto, embora não exista consenso entre os autores, a teoria da localização superficial do germe dentário, que predispõe à erupção dentária precoce, associada à hereditariedade, é a mais aceita ${ }^{(10,22)}$.

A erupção de dentes natais e neonatais, em geral, ocorre em crianças normais, com ou sem histórico familiar $^{(12,18)}$. Existem evidências de contribuição genética de algumas síndromes e anomalias de desenvolvimento 
associadas à presença de dentes natais e neonatais( ${ }^{(5)}$ - dentre elas, citam-se a displasia ectodérmica ${ }^{(26)}$, a síndrome de Pierre Robin ${ }^{(9)}$, os lábios fissurados ${ }^{(9,16)}$, a síndrome de Hallerman-Streiff ${ }^{(17,27)}$, a de Ellis-van Creveld ${ }^{(17,26)}$, a disostose craniofacial ${ }^{(21)}$, a paquioniquia congênita ${ }^{(21)}$ e a síndrome de Soto ${ }^{(23)}$.

\section{Características clínicas e radiográficas}

Os dentes natais e neonatais podem apresentar tamanho e forma normais; porém, na maioria das vezes, são pouco desenvolvidos, pequenos, cônicos, amarelados e hipoplásicos. Freqüentemente, mostram mobilidade acentuada, devido ao pobre desenvolvimento radicular, estando a parte coronária fixada pelos tecidos gengivais ${ }^{(3,12,28)}$. Radiograficamente, pode-se observar o grau de desenvolvimento radicular desses dentes e verificar se são supranumerários ou se fazem parte da dentição decídua ${ }^{(29,30)}$.

A classificação proposta por Massler e Savara ${ }^{(13)}$ está relacionada à época de erupção dentária dos dentes natais e neonatais, ou seja, dentes natais são aqueles observados na cavidade oral ao nascimento e dentes neonatais, aqueles que erupcionam durante os primeiros 30 dias de vida. Esta classificação não engloba características anatômicas, morfológicas e estruturais. A anomalia também foi classificada por Spouge e Feasby ${ }^{(4)}$, de acordo com o desenvolvimento: dente maturo quando o dente natal ou neonatal está completamente desenvolvido e apresentase com morfologia semelhante à do dente decíduo, com conseqüente bom prognóstico; e dente imaturo, quando este se apresenta com estruturas incompletas, sendo o prognóstico desfavorável. Segundo Kates et al ${ }^{(18)}$, dentes natais que permanecem na cavidade bucal por mais de quatro meses possuem bom prognóstico.

Outra classificação baseada nas características clínicas foi proposta por $\mathrm{To}^{(7)} \mathrm{e}$ consiste em quatro categorias:

1. coroa em forma de concha, com pobre inserção alveolar pelo tecido gengival e ausência radicular;

2. coroa sólida pobremente fixada ao alvéolo pelo tecido gengival e com desenvolvimento radicular ausente ou pequeno;

3. bordo incisal da coroa irrompido no tecido gengival;

4. tecido gengival edemaciado, com o dente não irrompido, porém palpável.

De acordo com o autor, os dentes natais que se enquadram na categoria 1 e 2, quando apresentam mobilidade acentuada, têm indicação de extração.

\section{Diagnóstico}

O correto diagnóstico é importante para definir se o dente natal ou neonatal faz parte da dentição decídua normal ou se é supranumerário, evitando-se, assim, extrações indiscriminadas, visto que a remoção prematura e iatrogênica desse dente pode causar perda de espaço e colapso dos arcos dentários em desenvolvimento, com conseqüente má-oclusão na dentição permanente ${ }^{(3,31)}$. O diagnóstico diferencial deve ser realizado por meio do exame radiográfico, uma vez que é difícil decidir com base somente na forma dentária ${ }^{(13)}$. Assim, quando o dente é supranumerário, observa-se radiograficamente um maior número de dentes em desenvolvimento, comparado ao dente natal ou neonatal pertencente à série normal.

Algumas manifestações bucais, como cistos da lâmina dentária e os nódulos de Bohn, comuns na cavidade oral de recém-nascidos, podem confundir o profissional quanto ao correto diagnóstico de dentes natais e neonatais. O diagnóstico diferencial destas alterações é realizado por meio de exame radiográfico ${ }^{(32)}$.

\section{Sintomas clínicos e complicações}

Devido à ausência de formação radicular, os dentes natais e neonatais apresentam grande mobilidade, podendo se mover em todas as direções e causar dor e desconforto ao bebê durante a amamentação, sendo tais sintomas comumente observados $^{(12,15)}$. Essa hipermobilidade é motivo de preocupação, pois a criança pode deglutir ou aspirar o dente ${ }^{(13,17,33)}$. Apesar de não haver descrições na literatura de casos de aspiração, este risco é iminente, diante de relatos de esfoliação espontânea desses dentes ${ }^{(12,23,24,34)}$.

Os dentes natais e neonatais também podem estar relacionados a sinais e sintomas característicos da época de erupção dos dentes decíduos da série normal, tais como diarréia, sialorréia, mal-estar, irritação e febre ${ }^{(35,36)}$. Entretanto, não existem evidências científicas que comprovem essa relação.

A ulceração traumática no ventre lingual, conhecida como doença de Riga-Fede, está freqüentemente associada à presença de dentes natais e neonatais em recém-nascidos e é causada pelos bordos incisais pontiagudos e cortantes. A ulceração tende a crescer de acordo com o trauma de repetição, podendo provocar uma fibrose local do tecido, com aparência de úlcera granulomatosa. Esta complicação dificulta a sucção e a alimentação do bebê, podendo causar deficiências nutricionais ${ }^{(37-40)}$.

A anomalia pode ainda causar lacerações ao seio materno $^{(12,15)}$; entretanto, Hals ${ }^{(24)}$, Zhu e King ${ }^{(5)}$ e Walter et al ${ }^{(32)}$ relataram não existir relação entre o ferimento no seio mater- 
no e a presença destes dentes, visto que, durante a sucção, a língua se interpõe entre o dente e o seio, ocorrendo somente uma lesão na base da língua do bebê.

Adicionalmente, tais dentes podem estar associados ao granuloma piogênico, que consiste em lesão sobressaliente de tecido conjuntivo, que ocorre em resposta a traumas ou irritações locais. Esta lesão normalmente se localiza na gengiva; porém, pode também estar presente em locais sujeitos a trauma, como língua, lábio e mucosa jugal ${ }^{(21)}$. Muench et al ${ }^{(41)}$ relataram o desenvolvimento de granuloma piogênico na região anterior da crista alveolar da mandíbula em um bebê de seis dias de idade, que apresentava dente natal. Estes autores sugerem que a extração desse dente tenha estimulado o desenvolvimento do granuloma piogênico, não existindo relatos anteriores similares.

Outra complicação relacionada a esta anomalia é o desenvolvimento de cárie precoce da infância, uma vez que os dentes natais e neonatais, além de apresentarem menor espessura de esmalte, podem ter deficiências na mineralização e sulcos ou rugosidades em sua superfície, predispondo à colonização por microrganismos cariogênicos ${ }^{(42)}$. Almeida et al ${ }^{(43)}$ observaram comprometimento endodôntico por cárie em dois dentes natais inferiores da série normal de um bebê de sete meses de idade. Devido ao fato de os elementos envolvidos gerarem dor, dificultando a alimentação do bebê e levando ao comprometimento do estado de saúde, a exodontia foi o tratamento de eleição. É importante que os profissionais da área de saúde orientem os responsáveis quanto à necessidade de higienização desses dentes, por serem imaturos e hipoplásicos, prevenindo o desenvolvimento de lesões de cárie.

Apesar de não existirem relatos na literatura de que os dentes natais e neonatais causem alterações de desenvolvimento nos dentes permanentes sucessores, Marcushamer $e t$ al ${ }^{(44)}$ descreveram dois casos de microdontia em incisivos centrais inferiores permanentes associados à história prévia de dentes natais na região correspondente.

\section{Medidas terapêuticas}

O estabelecimento do plano de tratamento é um desafio para o profissional. A decisão de manter ou não o dente na cavidade oral deve levar em consideração alguns fatores, tais como o grau de implantação e a mobilidade dentária, os problemas durante a sucção, a interferência com a amamentação, a possibilidade de lesão traumática e a dentição à qual o dente pertence: decídua normal ou supranumerária ${ }^{(3)}$.

Quando o dente pertencer à dentição normal, todos os fatores acima citados devem ser considerados. Se o dente apresentar uma boa implantação, a primeira opção de tratamento consistirá na manutenção do dente na cavidade bucal, desde que não cause prejuízos para o bebê $\hat{~}^{(17)}$. Entretanto, se estiver interferindo na amamentação ou apresentar grande mobilidade, com risco de deslocamento e aspiração, o dente deve ser removido ${ }^{(15,32)}$.

Se estes dentes causarem ulcerações na base da língua (doença de Riga-Fede) do bebê e no seio materno, pode-se indicar a extração $^{(24,28)}$. Para alguns autores ${ }^{(5,33)}$, a úlcera de Riga-Fede e o ferimento do seio materno durante a amamentação, por si só, não são indicações para a extração, visto que outra alternativa de tratamento consiste no alisamento dos bordos incisais ${ }^{(12)}$.

Se o tratamento de eleição for a extração do dente, algumas precauções devem ser tomadas. O procedimento cirúrgico deve ser evitado durante os primeiros dez dias de vida do recém-nascido, para prevenir hemorragias, considerando-se a incapacidade da flora intestinal da criança de produzir a vitamina $\mathrm{K}$ necessária para a produção de protrombina, que é imprescindível durante o processo de coagulação ${ }^{(28,33,45)}$. Desta forma, é mais seguro esperar de dez a 15 dias para realizar a extração.

Caso seja necessária a extração imediata do dente, o dentista deve solicitar uma avaliação pelo médico pediatra, para prescrição profilática de dose adequada de vitamina $\mathrm{K}$, que deverá ser administrada antes do procedimento cirúrgico, para que este seja realizado de forma segura ${ }^{(28,46)}$. Allwright ${ }^{(33)}$ relatou a ausência de episódios de hemorragia após a extração de 25 dentes natais e neonatais em 15 bebês, nos quais não foram tomadas medidas preventivas, pois as crianças tinham mais de 20 dias de vida.

Ryba e Kramer ${ }^{(47)}$, Southam ${ }^{(48)}$, Berendsen e Wakkerman ${ }^{(45)} \mathrm{e}$ Nedley et al ${ }^{(49)}$ relataram a formação de estruturas semelhantes a dentes após a extração de dentes natais ou neonatais, fato atribuído à permanência e crescimento de remanescentes da papila dentária. Esse fenômeno é raro, sendo indicada a extração dessas estruturas odontóides. Tsubone et al ${ }^{(50)}$ relataram a erupção de uma estrutura calcificada semelhante a um tecido ósseo seis meses após a esfoliação espontânea do incisivo central inferior neonatal, associado a um abscesso gengival. A análise histológica mostrou a presença de dentina, polpa, cemento e componentes semelhantes aos do ligamento periodontal. Dyment et al ${ }^{(34)}$ também descreveram o desenvolvimento de duas estruturas mineralizadas em criança de nove meses de idade, após a remoção de dentes neonatais ainda quando recém-nascida. Os autores recomendam a curetagem do alvéolo no momento da extração dentária, para prevenir o desenvolvimento dessas estruturas.

O procedimento de extração dentária, embora seja comumente executado pelos pediatras em alguns serviços de saúde, 
deve ser realizado pelo odontopediatra, uma vez que é necessária uma avaliação radiográfica criteriosa, a fim estabelecer a conduta adequada, pois o dente pode ou não pertencer à série normal. É importante que os pediatras encaminhem seus pacientes para uma consulta odontológica, com o objetivo de obter um tratamento seguro e proporcionar uma saúde global ao paciente ${ }^{(51)}$.

\section{Interação entre odontopediatras e pediatras}

A odontologia, cada vez mais, tem abordado o atendimento de bebês com o intuito de instituir precocemente medidas educativas e preventivas. Desta forma, é importante que exista uma interação entre odontopediatras e pediatras, para melhor orientar sobre as medidas de saúde aos responsáveis pela criança. Esse elo, entretanto, é pouco observado em nossa sociedade.

Em 1996, Schalka e Rodrigues ${ }^{(52)}$ observaram que apenas 10,4\% dos médicos pediatras incentivam a consulta odontológica logo após o início da erupção dentária ou até, no máximo, 18 meses de vida; 54,2\% indicam na faixa etária de dois a três anos; $10,4 \%$ entre quatro e cinco anos e $4,2 \%$ somente na fase escolar, por volta dos sete anos de idade.

Em um estudo realizado por Barroso et a $l^{(53)}$ em uma Unidade Básica de Saúde, no município de Niterói, Rio de Janeiro, das 100 gestantes entrevistadas, todas levaram seus filhos ao pediatra na primeira infância, enquanto 55\% levaram as crianças para a consulta odontológica. A maioria das mães (82\%) demonstrou estar orientada desde o pré-natal com relação à necessidade de prevenção de saúde geral de seus filhos, ao passo que, com relação à saúde bucal das crianças, somente $14 \%$ das entrevistadas receberam orientações para procurar o odontopediatra e $45 \%$ nunca levaram seus filhos ao dentista.

Em relação ao aprendizado sobre odontologia preventiva, foi observado por Schalka e Rodrigues ${ }^{(52)}$ que a maioria dos profissionais $(54,6 \%)$ nunca recebeu qualquer informação; $14,5 \%$ relataram ter recebido orientações durante o curso de graduação; 20,8\% durante a residência pediátrica e $22,9 \%$ em cursos extracurriculares, mostrando um baixo conhecimento dos médicos pediatras em relação à saúde bucal. Estes autores concluíram que se faz necessária a introdução de seminários e palestras sobre saúde bucal na formação do médico pediatra, para garantir uma rotina de condutas aos cuidados orais.

Freire $e t a l^{(54)}$, em um estudo realizado com 96 médicos pediatras do serviço público de Goiânia, Goiás, mostraram que $38,5 \%$ dos profissionais receberam informação sobre saúde bucal durante o curso de graduação, $28,1 \%$ em cursos de reciclagem fora do Sistema Único de Saúde e apenas 24\% durante a residência pediátrica. Quanto à auto-avaliação do nível de informação sobre saúde bucal, 24\% dos médicos o consideravam satisfatório; $59,4 \%$, relativamente satisfatório; e $6,7 \%$, não-satisfatório. Na opinião dos pesquisados, $67,7 \%$ acreditam que deva existir uma interação entre o cirurgião-dentista e o médico, visto que os primeiros são os profissionais responsáveis pela prevenção de cárie nos primeiros anos de vida.

Os pediatras, por serem os profissionais da área de saúde a ter contato com a criança desde o nascimento, são fundamentais no reconhecimento dos dentes natais e neonatais, devendo, desta forma, encaminhá-la ao odontopediatra para que a conduta terapêutica mais adequada seja instituída. Tal encaminhamento deve ocorrer antes da época de erupção dos dentes, para que o profissional oriente sobre dieta e higiene bucal adequadas, além de outras medidas preventivas. Assim, o profissional de odontologia pode auxiliar no diagnóstico de algumas doenças sistêmicas que apresentam suas manifestações mais precoces na cavidade bucal, enquanto o médico pediatra pode ajudar na prevenção de doenças bucais, orientando e encaminhando àquele profissional seus pacientes precocemente.

\section{Conclusões}

Os dentes natais e neonatais constituem-se em fatores de risco para o desenvolvimento da cárie dentária, da doença de Riga-Fede e de ulcerações no seio materno, além do risco de aspiração e de problemas nutricionais, como o comprometimento ao aleitamento materno. O pediatra, por ser o primeiro profissional da saúde a estabelecer contato com a criança, possui importante papel no encaminhamento desses pacientes para a avaliação odontopediátrica. Vale ressaltar que a responsabilidade não é só do odontopediatra, mas também do pediatra, em buscar conhecimentos sobre a seqüência normal do desenvolvimento dentário em ambas as dentições, sabendo reconhecer os desvios de erupção e os possíveis danos que estas alterações podem causar. Portanto, é de grande valia a introdução de seminários e palestras sobre saúde bucal na formação do médico pediatra para garantir uma rotina de condutas aos cuidados orais.

Assim, é importante que exista uma interação entre odontopediatras e pediatras, pois esta permite um melhor acompanhamento do crescimento e do desenvolvimento da criança, proporcionando o diagnóstico precoce e a abordagem integral da criança, com vista à promoção de saúde. 


\section{Referências bibliográficas}

1. Lunt RC, Law DB. A review of the chronology of eruption of deciduous teeth. J Am Dent Assoc 1974;89:872-9.

2. Delbem AC, Faraco Júnior IM, Percinoto C. Natal teeth: case report. J Clin Pediatr Dent 1996;20:325-7.

3. Cunha RF, Boer FA, Torriani DD, Frossard WT. Natal and neonatal teeth: review of the literature. Pediatr Dent 2001;23:158-62.

4. Spouge JD, Feasby WH. Erupted teeth in the newborn. Oral Surg Oral Med Oral Pathol 1966;22:198-208.

5. Zhu J, King D. Natal and neonatal teeth. ASDC J Dent Child 1995;62:123-8.

6. Nik-Hussein NN. Natal and neonatal teeth. J Pedod 1990;14:110-2.

7. To EW. A study of natal teeth in Hong Kong Chinese. Int J Paediatr Dent 1991;1:73-6.

8. Long SM, Chelotti A, Rego MA, Jorge AO. Dente natal: relato de caso clínico. Rev Assoc Paul Cir Dent 1994;48:1291-4.

9. Leung AK. Natal teeth. Am J Dis Child 1986;140:249-51.

10. Bodenhoff J, Gorlin RJ. Natal and neonatal teeth; folklore and fact. Pediatrics 1963;32:1087-93.

11. Epstein IR. Natal tooth causing ulceration of the tongue. J Dent Assoc S Afr 1963;18:179

12. Martínez-Gómez V. Dientes natales y neonatales - revision de la literatura. Acta Odontol Venez 1997;35:54-60.

13. Massler M, Savara BS. Natal and neonatal teeth; a review of 24 cases reported in the literature. J Pediatr 1950;36:349-59.

14. Anderson RA. Natal and neonatal teeth: histologic investigation of two black females. ASDC J Dent Child 1982;49:300-3.

15. Leung AK, Robson WL. Natal teeth: a review. J Natl Med Assoc 2006;98:226-8.

16. de Almeida CM, Gomide MR. Prevalence of natal/neonatal teeth in cleft lip and palate infants. Cleft Palate Craniofac J 1996;33:297-9.

17. Chow MH. Natal and neonatal teeth. J Am Dent Assoc 1980;100:215-6.

18. Kates GA, Needleman HL, Holmes LB. Natal and neonatal teeth: a clinical study. J Am Dent Assoc 1984;109:441-3.

19. Gorlin RJ, Goldman HM, Thoma K. Patologia oral. $4^{\text {th }}$ ed. Barcelona: Salvatore; 1973.

20. Boyd JD, Miles AE. An erupted tooth in a cyclops foetus. Br Dent J 1951;91:173-81.

21. Shafer WG, Hine MK, Levy BM. Tratado de patologia bucal. $4^{a}$ ed. Rio de Janeiro: Guanabara; 1985.

22. Leung AK. Management of natal teeth. J Am Dent Assoc 1987;114:762.

23. Bigeard L, Hemmerle J, Sommermater JI. Clinical and ultrastructural study of the natal tooth: enamel and dentin assessments. ASDC J Dent Child 1996;63:23-31.

24. Hals E. Natal and neonatal teeth; histologic investigations in two brothers. Oral Surg Oral Med Oral Pathol 1957;10:509-21.

25. Bodenhoff J. Natal and neonatal teeth. Dent Abstr 1960;5:485-6.

26. Feingold M. Ellis-van Creveld syndrome. Clin Pediatr (Phila) 1966;5:431-6.

27. da Fonseca MA, Mueller WA. Hallerman-Streiff syndrome: case report and recommendations for dental care. ASDC J Dent Child 1994;61:334-7.

28. Rusmah M. Natal and neonatal teeth: a clinical and histological study. J Clin Pediatr Dent 1991;15:251-3.

29. Brandt SK, Shapiro SD, Kittle PE. Immature primary molar in the newborn. Pediatr Dent 1983:5:210-3.

30. Ronk SL. Multiple immature teeth in a newborn. J Pedod 1982;6:254-60.
31. Hebling J, Zuanon AC, Vianna DR. Dente natal. Odontol Clin 1997;7:37-40.

32. Walter LRF, Ferelle A, Issao M. Odontologia para o bebê. São Paulo: Artes Médicas; 1996.

33. Allwright WC. Natal and neonatal teeth: a study among Chinese in Hong Kong Br Dent J 1958;105:163-72.

34. Dyment $\mathrm{H}$, Anderson R, Humphrey J, Chase I. Residual neonatal teeth: a case report. J Can Dent Assoc 2005;71:394-7.

35. King DL. Teething revisited. Pediatr Dent 1994;16:179-82.

36. Diniz MB, Bolini PDA, Minarelli-Gaspar AM. Sintomas locais e sistêmicos relacionados à erupção de dentes decíduos. Rev Paul Pediatr 2006;24:71-7.

37. Zaenglein AL, Chang MW, Meehan SA, Axelrod FB, Orlow SJ. Extensive RigaFede disease of the lip and tongue. J Am Acad Dermatol 2002;47:445-7.

38. Hegde RJ. Sublingual traumatic ulceration due to neonatal teeth (Riga-Fede disease). J Indian Soc Pedod Prev Dent 2005;23:51-2.

39. Baroni A, Capristo C, Rossiello L, Faccenda F, Satriano RA. Lingual traumatic ulceration (Riga-Fede disease). Int J Dermatol 2006;45:1096-7.

40. Campos-Muñoz L, Quesada-Cortés A, Corral-De la Calle M, Arranz-Sánchez D, Gonzalez-Beato MJ, De Lucas R et al. Tongue ulcer in a child: Riga-Fede disease. J Eur Acad Dermatol Venereol 2006;20:1357-9.

41. Muench MG, Layton S, Wright JM. Pyogenic granuloma associated with a natal tooth: case report. Pediatr Dent 1992;14:265-7.

42. Tinanoff N, Daley NS, O'Sullivan DM, Douglass JM. Failure of intense preventive efforts to arrest early childhood and rampant caries: three case reports. Pediatr Dent 1999;21:160-3.

43. Almeida ML, Damasceno LM, Portela MB, Primo LG. Dente natal: fator de risco para o desenvolvimento de cárie em bebês. Rev Paul Pediatr 2001;19:202-6.

44. Marcushamer M, King DL, McCourt JW Jr. Microdontic teeth succedaneous to natal teeth: a report of two cases. Pediatr Dent 1992;14:400-1.

45. Berendsen WJ, Wakkerman HL. Continued growth of the dentinal papillae after extraction of neonatal teeth: report of case. ASDC J Dent Child 1988;55:139-41.

46. Holder TM, Ashcraft KW. Pediatric surgery. Philadelphia: WB Saunders; 1980.

47. Ryba GE, Kramer IR. Continued growth of human dentine papillae following removal of the crowns of partly formed deciduous teeth. Oral Surg Oral Med Oral Pathol 1962;15:867-75.

48. Southam JC. Retained dentine papillae in the newborn. A clinical and histopathological study. Br Dent J 1968;125:534-8.

49. Nedley MP, Stanley RT, Cohen DM. Extraction of natal and neonatal teeth can leave odontogenic remnants. Pediatr Dent 1995;17:457.

50. Tsubone H, Onishi T, Hayashibara T, Sobue S, Ooshima T. Clinico-pathological aspects of a residual natal tooth: a case report. J Oral Pathol Med 2002;31:239-41.

51. Silva Neto AR, Nascimento LG, Oliveira RG, Novaes LH. Dente Natal. Rev Paul Pediatr 1999;17:190-2.

52. Schalka MM, Rodrigues CR. A importância do médico pediatra na promoção da saúde bucal. Rev Saúde Pública 1996;30:179-86.

53. Barroso SP, Miasato JM, Graça TC. Avaliação da freqüência de visitas ao Pediatra $\mathrm{x}$ visitas ao Odontopediatra em Unidade Básica de Saúde do Município de Niterói-RJ. J Bras Odontoped Odontol Bebe 2000;3:324-7.

54. Freire MC, Macêdo RA, Silva WH. Conhecimentos, atitudes e práticas dos médicos pediatras em relação à saúde bucal. Pesqui Odontol Bras 2000;14:39-45. 